\title{
Increased expression of CD25, CD83, and CD86, and secretion of IL-12, IL-23, and IL-10 by human dendritic cells incubated in the presence of Toll-like receptor 2 ligands and Giardia duodenalis
}

\author{
Janine Obendorf ${ }^{1}$, Pablo Renner Viveros ${ }^{1}$, Michael Fehlings ${ }^{1}$, Christian Klotz $^{2}$, Toni Aebischer ${ }^{2}$ and Ralf Ignatius ${ }^{1 *}$
}

\begin{abstract}
Background: Effects of Giardia duodenalis on dendritic cell (DC) functions may contribute to the pathogenesis of chronic giardiasis. G. duodenalis lysate has been shown to inhibit the activation of murine DCs through the ligands of various Toll-like receptors (TLRs), including TLR2 and TLR4. Our study aimed at translating these findings to human DCs.

Findings: As described previously for murine DCs, also human DCs were only weakly activated by the parasite itself. LPS-stimulated DCs incubated in the presence of $G$. duodenalis lysate produced less IL-12/23p40 ( $p=0.002)$, IL-12p70 ( $p=0.011)$, and IL-23 ( $p=0.004)$, but more IL-10 ( $p=0.006)$ than cells incubated in the absence of the parasite. Concomitantly, the expression of CD25, CD83, CD86, and HLA-DR was reduced on G. duodenalis-incubated DCs as compared to control cells. In contrast, human DCs stimulated through TLR2 in combination with TLR1 or TLR6 and G. duodenalis lysate secreted significantly more IL-12/23p40 ( $p=0.006), I L-23(p=0.002)$, and IL-10 ( $p=0.014)$ than cells stimulated through TLR2 ligands alone. Ligands for TLR2/TLR1 or TLR2/TLR6 also induced enhanced extracellular expression of CD25, CD83, and CD86 ( $p<0.05)$.

Conclusions: In contrast to murine DCs, human DCs incubated in the presence of G. duodenalis and stimulated through TLR2 show increased activation as compared to cells incubated in the absence of the parasite. Thus, TLR2 ligands, e.g., delivered by probiotic lactobacilli, might be beneficial in human giardiasis through an adjuvant effect on the induction of cellular immune responses against G. duodenalis.
\end{abstract}

Keywords: Dendritic cells, Giardia duodenalis, Toll-like receptors, CD25, CD83, CD86, IL-12, IL-23, IL-10

\section{Findings}

\section{Background}

Giardia duodenalis is the most frequent parasitic agent of gastroenteritis worldwide and has been targeted as part of the WHO "Neglected Disease Initiative" since 2006 [1]. In East Africa, for example, we and others have observed prevalences of $>60 \%$ in children younger than five years $[2,3]$. Notably, chronic (or recurrent) giardiasis in children has been associated with malnutrition, wasting, and stunting as well as reduced cognitive functions at a later age [4].

\footnotetext{
*Correspondence: ralf.ignatius@charite.de

${ }^{1}$ Institute of Tropical Medicine and International Health, Charité -

Universitätsmedizin Berlin, Spandauer Damm 130, Berlin 14050, Germany

Full list of author information is available at the end of the article
}

The pathogenesis of chronic giardiasis is poorly understood and both parasitic and host factors might be involved. The parasite is non-invasive but stays in the intestinal lumen where it adheres to epithelial cells. Since $\mathrm{CD}^{+} \mathrm{T}$ helper cells are involved in protection against G. duodenalis [5], chronic infection might result from insufficient antigen presentation that does not lead to the development of protective cellular immunity. Dendritic cells (DCs) might be critically involved in the induction of anti-Giardia immune responses as they are able to reach with their cellular protrusions into the gut lumen and sample antigen [6]. Following antigen-uptake, DCs may process and present specific peptides to naïve $\mathrm{T}$ lymphocytes, and DCs activated through ligands of 
pattern-recognition receptors (PRRs), e.g., Toll-like receptors (TLRs), C-type lectins, and nucleotide-binding oligomerization domain (NOD)-like receptors (NLRs), are essential for the induction of specific cellular immunity [7]. A recently published study with murine DCs has shown that G. duodenalis lysate may interfere with the activation of DCs through TLR2 or TLR4 in vitro [8]. Signals through both receptors might be relevant for the induction of anti-Giardia immune responses because G. duodenalis by itself only weakly activates murine DCs [8]. In vivo, bacteria belonging to the gut flora might deliver the required stimuli to $G$. duodenalis-processing DCs since TLR2 may recognize lipoproteins derived from Gram-positive while TLR4 binds LPS of Gramnegative bacteria [9].

The present study aimed at translating the previous findings from murine to human DCs. To monitor DC activation, we focused on the expression of typical surface markers and cytokine production [10]. Regarding cytokines, we analyzed the secretion of members of the IL-12 family. IL-12 is a pro-inflammatory cytokine, which consists in its biologically active form, IL-12p70, of a light chain (IL-12p35) and a heavy chain (IL-12p40), and favors the differentiation of Th1 cells [11]. IL-12p40 (together with a p19 subunit) may also be part of IL-23, which drives the development of $\mathrm{T}$ helper cells that secrete IL-17 (Th17) [12]. In addition, we analyzed the secretion of the anti-inflammatory cytokine, IL-10.

\section{Methods}

\section{Preparation of $G$. duodenalis lysate}

G. duodenalis strain WB-C6 (ATCC 50803) trophozoites were propagated in TYI-S-33 medium as previously described [13]. Parasites were harvested and washed by centrifugation through PBS. Pellets were resuspended in PBS, and the parasites counted and diluted to a concentration of $10^{8}$ parasites $/ \mathrm{ml}$. Lysates were prepared by subjecting the suspensions in three consecutive freezingthawing cycles.

\section{Generation of human DCs}

DCs were generated as described previously [14]. Briefly, peripheral blood mononuclear cells were isolated from buffy coats obtained from healthy donors (German Red Cross, Berlin). CD14 $4^{+}$monocytes were separated with magnetic microbeads (Miltenyi Biotec, Bergisch-Gladbach, Germany) and cultured at $3 \times 10^{6}$ cells/well in 6-well cell culture dishes (Nunc, Roskilde, Denmark) for six days in medium consisting of RPMI 1640 supplemented with $2 \mathrm{mM}$ L-glutamine, $10 \mathrm{mM}$ HEPES, penicillin $(1,000 \mathrm{U} / \mathrm{ml})$ streptomycin $(1,000 \mu \mathrm{g} / \mathrm{ml})$ (all from Gibco, Invitrogen, Karlsruhe, Germany), $50 \mu \mathrm{M}$ 2-mercaptoethanol (Sigma, Taufkirchen, Germany), 10\% fetal calf serum (Biochrom, Berlin, Germany), $1000 \mathrm{U} / \mathrm{ml}$ human rGM-
CSF (sargramostim, Leukine ${ }^{\oplus}$, Berlex, Richmond, CA, USA), and $100 \mathrm{U} / \mathrm{ml}$ human rIL-4 (R\&D Systems, Wiesbaden-Nordenstadt, Germany). On days 2 and 4, fresh medium and cytokines were added to the wells.

\section{Incubation of human DCs with G. duodenalis lysate and TLR ligands}

On day 6, DCs were harvested and transferred to 96-well round-bottom trays (Nunc) at $10^{5}$ cells/well. The TLR stimuli Pam2CSK4 and Pam3CSK4 (100 ng/ml; Invivogen, Toulouse, France) or LPS $(1 \mu \mathrm{g} / \mathrm{ml}$; derived from Escherichia coli 0111:B4, Sigma) were added and the cells incubated in the presence or absence of G. duodenalis lysate (equivalent to five trophozoites/cell). Immature cells kept in medium were equally incubated in the presence or absence of G. duodenalis lysate.

\section{Flow cytometric analyses}

The phenotype of DCs was monitored by flow cytometry with PE- or FITC-labeled anti-human mAbs against HLA-DR, CD14, CD25, CD86 (all BD Pharmingen), CD83 (Caltag Laboratories, Hamburg, Germany), or the appropriate isotype controls, as previously described [14]. Cells were fixed with $10 \%$ formalin/PBS (v/v) before analysis on a FACScan ${ }^{\circledR}$ cytometer with CELLQuest ${ }^{\oplus}$ Pro software (BD Pharmingen). Percentages of positive cells and median fluorescence intensities (MFIs) were calculated.

\section{Analysis of cytokine secretion by DCs}

Cell-free supernatants of cell cultures were harvested after $48 \mathrm{~h}$ and stored at $-80^{\circ} \mathrm{C}$ until analysis by sandwich ELISAs for IL-12p40, IL-12p70, and IL-10 (all U-CyTech, Utrecht, The Netherlands), or IL-23 (eBioscience). Samples with known cytokine concentrations provided by the manufacturers of the ELISA kits were included in each analysis and used to create standard curves. Only values within the linear section of these curves were considered. The level of detection was $5 \mathrm{pg} / \mathrm{ml}$ for all assays.

\section{Statistical analyses}

Data were analyzed statistically with the nonparametric Wilcoxon matched-pairs signed rank test using GraphPad Prism version 6.0a for Mac OS X. Differences were considered statistically significant for $\mathrm{p}<0.05$.

\section{Results}

We incubated human immature monocyte-derived DCs in the presence or absence of G. duodenalis lysate with the TLR4 ligand LPS or with ligands binding to TLR2/TLR1 or TLR2/TLR6, i.e., Pam3CSK4 and Pam2CSK4, respectively, since TLR2 is expressed together with either TLR1 or TLR6 [9]. Control cells were kept in the absence of 
TLR ligands. Two days later, supernatants were collected and the cells harvested.

Flow cytometry revealed that similar to murine DCs, G. duodenalis lysate by itself only weakly activated human DCs (data not shown). On LPS-activated DCs, it reduced the expression of CD25 and CD83, typical DC surface markers indicative of cellular activation, and of CD86 and HLA-DR, which are involved in antigen-presentation (Table 1). Similarly, the MFIs for CD83 and CD86 were reduced on G. duodenalis-incubated DCs as compared to control cells (data not shown). In contrast, among DCs stimulated through either TLR2/TLR1 or TLR2/TLR6 and incubated in the presence of $G$. duodenalis lysate, significantly more cells expressed CD25, CD83, and CD86 than in DC populations incubated in the absence of the parasite-derived stimuli (Table 1). These larger numbers of CD25 and CD83 positive cells also correlated with significantly higher MFIs for these molecules (data not shown).

We next determined the concentrations of IL-12/ 23p40, IL-12p70, IL-23, and IL-10 in all supernatants and again observed distinct effects of $G$. duodenalis lysate dependent on the TLR agonist involved. When cells were activated through TLR4, G. duodenalis significantly reduced the production of IL-12/23p40, IL-12p70, and IL-23 while the IL-10 secretion was enhanced (Figure 1). In contrast to these findings and those previously obtained with murine DCs, exposure to G. duodenalis lysate enhanced the secretion of IL-12/23p40, IL-23, and IL-10 by DCs activated through TLR2 - although the cells produced comparably less cytokines than LPSstimulated DCs (Figure 1). The IL-12p70 concentrations of TLR2-activated DCs were below the level of detection for most samples, irrespective of the presence or absence of $G$. duodenalis lysate in the cell cultures. As noted before for LPS-stimulated human monocytes [15], we also observed inter-individual differences, i.e., low and high responders, in the cytokine responses upon TLR-ligation of the DCs. The discordant values, however, did not affect the statistical analyses of the data.

Thus, while we could translate the previous murine data regarding the inhibition of LPS-stimulated DCs through G. duodenalis to the human cell system, we observed the opposite for DCs stimulated through TLR2. Exposure of TLR2-activated DCs to G. duodenalis considerably enhanced the expression of crucial surface molecules and the secretion of cytokines belonging to the IL-12 family.

\section{Conclusion}

In contrast to TLR4 ligands and in contrast to murine DCs, TLR2 ligands may augment the antigen-presenting functions of human DCs in giardiasis. Although the LPS-induced cytokine responses were comparably stronger than those induced by the TLR2 ligands, which might be due to the concentrations of the stimuli used, we feel that the data together, i.e., opposite results for cytokine secretion and also surface molecule expression for the stimuli tested, may argue for an additive effect of TLR2 and G. duodenalis on human DC functions. In contrast, G. duodenalis apparently may interfere with immune responses induced by human DCs activated through TLR4 agonists.

Probiotic Lactobacillus spp. are recognized by human DCs through TLR2 and NOD2 [16], and may induce dependent on TLR2 - the secretion of IL-12, IL-10, and type I interferons as shown for murine DCs [17]. In a Gerbil model, interestingly, lactobacilli interfere with G. duodenalis infection in vivo [18], and this effect could partly depend on TLR2 signaling. Although this remains to be investigated, our demonstration that G. duodenalis can augment TLR2-mediated DC function suggests that TLR2 ligands might be of considerable interest in the management of clinical giardiasis. Since an anti-giardial effect by Lactobacillus spp. on murine giardiasis is also evident when the bacteria are administered together with anti-giardial drugs [19] or in malnourished animals [20], clinical studies are warranted in which the potential benefit of lactobacilli in the treatment of human giardiasis patients may be evaluated as a combination of bacteria with standard therapy, e.g., metronidazole or tinidazole, but also in therapy-refractory chronic giardiasis.

Table 1 DC activation markers expressed by human DCs incubated in the presence of ligands for TLR4, TLR2/TLR1, or TLR2/TLR6, and in the presence or absence of G. duodenalis lysate

\begin{tabular}{ccccccc}
\hline Molecule & LPS & LPS + Gd & Pam3CSK & Pam3CSK + Gd & Pam2CSK4 & Pam2CSK4 + Gd \\
\hline CD25 & $84[73,90]^{*}$ & $82[66,88]^{*}$ & $8[3,21]$ & $20[7,30]^{*}$ & $10[3,27]$ & $21[9,35]^{*}$ \\
CD83 & $90[85,95]$ & $84[80,93]^{*}$ & $18[12,51]$ & $35[22,59]^{*}$ & $23[15,51]$ & $39[24,63]^{*}$ \\
CD86 & $98[97,99]$ & $96[93,97]^{*}$ & $51[48,61]$ & $67[59,79]^{*}$ & $56[48,71]$ & $70[61,86]^{*}$ \\
HLA-DR & $99[98,99]$ & $97[95,98]^{*}$ & $88[84,97]$ & $90[86,97]$ & $88[84,96]$ & $92[84,97]$
\end{tabular}

\#Median percentage of positive cells ( $25 \%$ and $75 \%$ percentiles given in square brackets) obtained with cells from ten donors, except for CD86, which could be analyzed for eight donors only (data of isotype controls were subtracted).

${ }^{*} \mathrm{p}<0.05$ compared to DCs incubated in the presence of LPS, Pam3CSK, or Pam2CSK4 but in the absence of G. duodenalis (Wilcoxon matched-pairs signed rank test). 

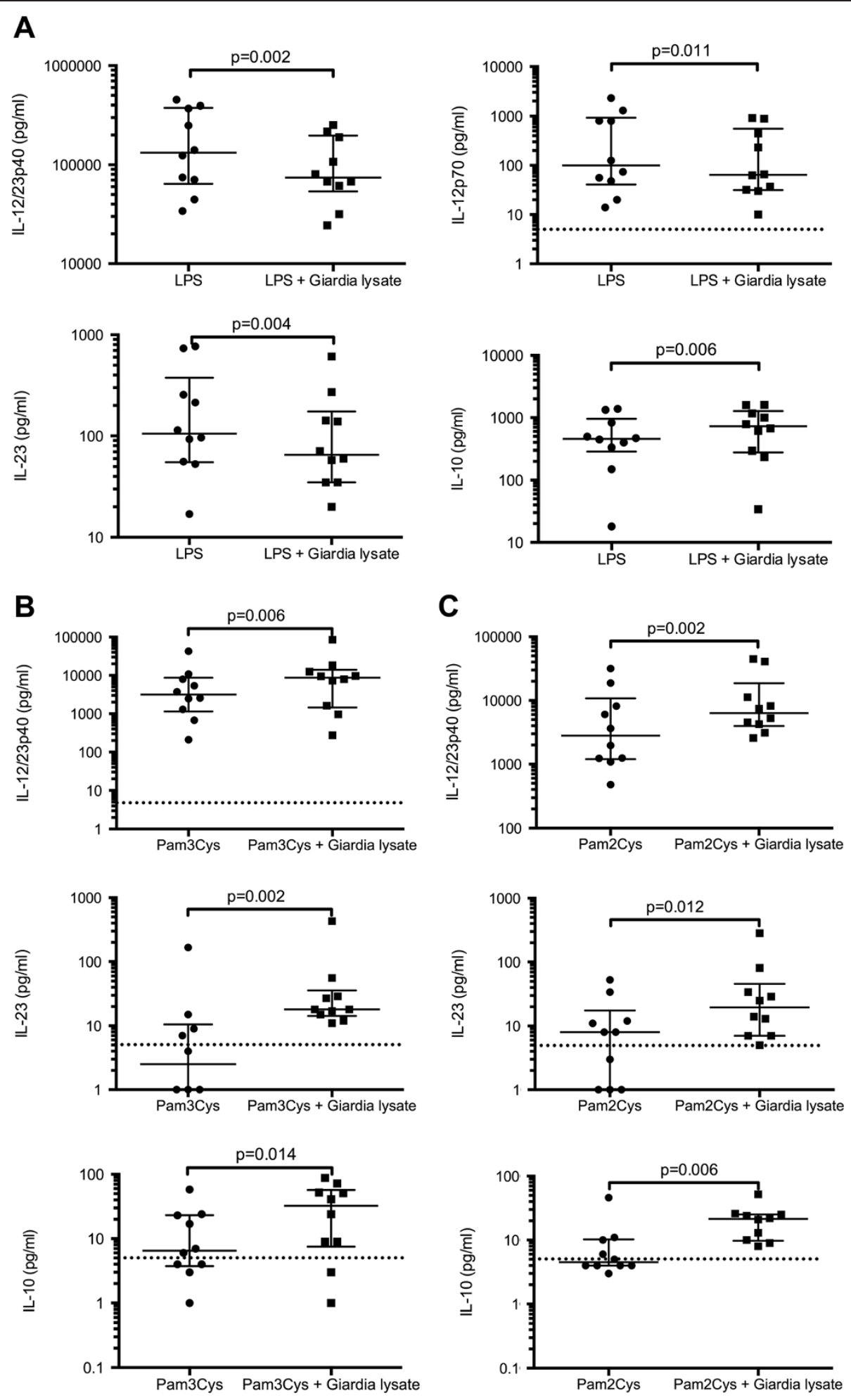

Figure 1 TLR2 ligands but not LPS augment the secretion of IL-12 cytokines by human DCs incubated in the presence of G. duodenalis. Immature DCs were incubated with (A) LPS (1 $\mu \mathrm{g} / \mathrm{ml})$, (B) Pam3CSK4 (100 ng/ml), or (C) Pam2CSK4 (100 ng/ml) in the presence or absence of $G$. duodenalis lysate (equivalent to five trophozoites/cell). Two days later, cell-free supernatants were collected, stored at $-80^{\circ} \mathrm{C}$, and the cytokine concentrations determined by ELISA. Median plus 25\% and 75\% percentiles for cells from ten donors. Dotted horizontal lines indicate the level of detection of the ELISA assays $(5 \mathrm{pg} / \mathrm{ml})$. 


\section{Competing interests}

The authors declare that they have no competing interests.

\section{Authors' contributions}

$\mathrm{JO}$ and PRV performed the experiments, analyzed the data, and wrote the manuscript; MF analyzed the data and wrote the manuscript; CK and TA prepared the G. duodenalis lysate, designed the study, and wrote the manuscript; RI designed and supervised the study and wrote the manuscript. All authors have approved the final version of the manuscript.

\section{Acknowledgements}

We thank Petra Gosten-Heinrich for excellent technical assistance. This study was supported by the Charité - Universitätsmedizin Berlin and in part by a grant to T.A. (01Kl1019) from the National Research Platform for Zoonosis, which is funded by the German Federal Ministry of Education and Research (BMBF).

\section{Author details}

${ }^{1}$ Institute of Tropical Medicine and International Health, Charité Universitätsmedizin Berlin, Spandauer Damm 130, Berlin 14050, Germany. ${ }^{2}$ Mycotic and Parasitic Agents and Mycobacteria, Department of Infectious Diseases, Robert Koch-Institute, Nordufer 20, Berlin 13353, Germany.

Received: 9 October 2013 Accepted: 1 November 2013

Published: 4 November 2013

\section{References}

1. Savioli L, Smith H, Thompson A: Giardia and Cryptosporidium join the 'Neglected Diseases Initiative'. Trends Parasitol 2006, 22(5):203-208.

2. Johnston AR, Gillespie TR, Rwego IB, McLachlan TL, Kent AD, Goldberg TL: Molecular epidemiology of cross-species Giardia duodenalis transmission in western Uganda. PLoS Negl Trop Dis 2010, 4(5):e683.

3. Ignatius $R$, Gahutu JB, Klotz C, Steininger C, Shyirambere C, Lyng M, Musemakweri A, Aebischer T, Martus P, Harms G, et al: High prevalence of Giardia duodenalis assemblage B infection and association with underweight in Rwandan Children. PLoS Negl Trop Dis 2012, 6(6):e1677.

4. Berkman DS, Lescano AG, Gilman RH, Lopez SL, Black MM: Effects of stunting, diarrhoeal disease, and parasitic infection during infancy on cognition in late childhood: a follow-up study. Lancet 2002, 359(9306):564-571.

5. Singer SM, Nash TE: T-cell-dependent control of acute Giardia lamblia infections in mice. Infect Immun 2000, 68(1):170-175.

6. Rescigno M, Urbano M, Valzasina B, Francolini M, Rotta G, Bonasio R, Granucci F, Kraehenbuhl JP, Ricciardi-Castagnoli P: Dendritic cells express tight junction proteins and penetrate gut epithelial monolayers to sample bacteria. Nat Immunol 2001, 2(4):361-367.

7. Ueno H, Klechevsky E, Morita R, Aspord C, Cao T, Matsui T, Di Pucchio T, Connolly J, Fay JW, Pascual V, et al: Dendritic cell subsets in health and disease. Immunol Rev 2007, 219:118-142.

8. Kamda JD, Singer SM: Phosphoinositide 3-kinase-dependent inhibition of dendritic cell interleukin-12 production by Giardia lamblia. Infect Immun 2009, 77(2):685-693.

9. Kawai T, Akira S: Toll-like receptors and their crosstalk with other innate receptors in infection and immunity. Immunity 2011, 34(5):637-650.

10. Steinman RM: Decisions about dendritic cells: past, present, and future. Ann Rev Immunol 2012, 30:1-22.

11. Trinchieri G: Interleukin-12 and the regulation of innate resistance and adaptive immunity. Nat Rev Immunol 2003, 3(2):133-146.

12. Aggarwal S, Ghilardi N, Xie MH, De Sauvage FJ, Gurney AL: Interleukin-23 promotes a distinct CD4 T cell activation state characterized by the production of interleukin-17. J Biol Chem 2003, 278(3):1910-1914.

13. Keister DB: Axenic culture of Giardia lamblia in TYI-S-33 medium supplemented with bile. Trans R Soc Trop Med Hyg 1983, 77(4):487-488.

14. Jasny E, Eisenblatter M, Matz-Rensing K, Tenner-Racz K, Tenbusch M, Schrod A, Stahl-Hennig C, Moos V, Schneider T, Racz P, et al: IL-12-impaired and IL-12-secreting dendritic cells produce IL-23 upon CD154 restimulation. J Immunol 2008, 180(10):6629-6639.

15. Schraut W, Wendelgass P, Calzada-Wack JC, Frankenberger M, Ziegler-Heitbrock HW: TNF gene expression in monocytes of low and high responder individuals. Cytokine 1997, 9(3):206-211.

16. Zeuthen LH, Fink LN, Frokiaer H: Toll-like receptor 2 and nucleotide-binding oligomerization domain-2 play divergent roles in the recognition of gut-derived lactobacilli and bifidobacteria in dendritic cells. Immunology 2008, 124(4):489-502.

17. Weiss $G$, Rasmussen $S$, Zeuthen $L H$, Nielsen BN, Jarmer $H$, Jespersen $L$, Frokiaer $\mathrm{H}$ : Lactobacillus acidophilus induces virus immune defence genes in murine dendritic cells by a Toll-like receptor-2-dependent mechanism. Immunology 2010, 131(2):268-281.

18. Humen MA, De Antoni GL, Benyacoub J, Costas ME, Cardozo Ml, Kozubsky L, Saudan KY, Boenzli-Bruand A, Blum S, Schiffrin EJ, et al: Lactobacillus johnsonii La1 antagonizes Giardia intestinalis in vivo. Infect Immun 2005, 73(2):1265-1269.

19. Shukla G, Kaur H, Sharma L: Comparative therapeutic effect of probiotic Lactobacillus casei alone and in conjunction with antiprotozoal drugs in murine giardiasis. Parasitol Res 2013, 112(6):2143-2149.

20. Shukla G, Sidhu RK: Lactobacillus casei as a probiotic in malnourished Giardia lamblia-infected mice: a biochemical and histopathological study. Can J Microbiol 2011, 57(2):127-135.

doi:10.1186/1756-3305-6-317

Cite this article as: Obendorf et al:: Increased expression of CD25, CD83, and CD86, and secretion of IL-12, IL-23, and IL-10 by human dendritic cells incubated in the presence of Toll-like receptor 2 ligands and Giardia duodenalis. Parasites \& Vectors 2013 6:317.

\section{Submit your next manuscript to BioMed Central and take full advantage of:}

- Convenient online submission

- Thorough peer review

- No space constraints or color figure charges

- Immediate publication on acceptance

- Inclusion in PubMed, CAS, Scopus and Google Scholar

- Research which is freely available for redistribution

Submit your manuscript at www.biomedcentral.com/submit
C BioMed Central 